\title{
ARTICLE \\ Low-cost and cleanroom-free fabrication of microneedles
}

\author{
Hojatollah Rezaei Nejad ${ }^{1, *}$, Aydin Sadeqi ${ }^{1, *}$, Gita Kiaee $^{2}$ and Sameer Sonkusale ${ }^{1}$
}

We present a facile, low-cost and cleanroom-free technique for the fabrication of microneedles using molds created by laser ablation. Microneedle mold with high aspect ratios is achieved on acrylic sheet by engraving a specific pattern of crossover lines $(\mathrm{COL})$ using $\mathrm{CO}_{2}$ laser cutter. Ablating $\mathrm{COL}$ pattern on the acrylic sheet creates a sharp conical shape in the center of the design. We have shown that a variety of microneedle shapes with different heights and tip angles can be easily achieved by changing the number and the length of the COL. Polydimethylsiloxane (PDMS) microneedles were fabricated by casting the PDMS on the mold. The resulted PDMS microneedles are oxygen plasma treated and then silanized. Another PDMS layer is casted on PDMS microneedles and detached after curing. The silanization prevents those two layers of PDMS from bonding to each other and makes them easily detachable. After detachment of the PDMS mold of microneedles, the mold is used to fabricate degradable polyvinyl alcohol microneedle patch suitable for transdermal drug delivery. The release kinetics of the needles are also shown and discussed in order to prove the applicability of the needles.

Keywords: drug delivery; laser ablation; microneedles

Microsystems \& Nanoengineering (2018) 4, 17073; doi:10.1038/micronano.2017.73; Published online: 15 January 2018

\section{INTRODUCTION}

Skin is considered as the largest organ in the body with around $1.5 \mathrm{~m}^{2}$ surface area in adults, and it can be a good administration route for drugs ${ }^{1}$. However, the outermost layer of skin, stratum corneum, which protects the human body from toxic chemicals, makes it challenging for high molecular weight and hydrophilic molecules to pass through this membrane ${ }^{2}$. Among different approaches to enhance the transdermal drug delivery, use of microneedles is a promising non-invasive approach to increase the permeability of the skin to drugs ${ }^{3}$. Microneedles create microchannels in the skin, which allows drug molecules to transport easily through them ${ }^{4}$. Different studies have shown that intradermal administration of specific drugs using microneedles increases the therapeutic efficacy compared to the intramuscular and the subcutaneous routes ${ }^{5}$. As reported previously, the dissolving microneedle patch can rapidly release the drug in skin; this is an efficient way to deliver large molecules like insulin ${ }^{6}$. Thus, microneedles provide easier route for transdermal drug delivery for molecules that are not readily absorbed topically. The main goal in the fabrication of microneedle is to achieve uniformity and reproducibility of the needle geometry at micron scale resolution to facilitate easy penetration of the needles in the skin. Microfabrication techniques using standard lithography are very promising in this regard. However, fabrication of microneedles has been shown to be very challenging mainly due to the threedimensional (3D) conical geometry and the high aspect ratio structures of the microneedles (the height of microneedles varies from 0.5 to $3 \mathrm{~mm}$ ). Microneedles are fabricated most commonly using molding methods ${ }^{7}$, where the molds are prepared using complicated microfabrication procedures in the cleanroom. These procedures include photolithography using deep X-ray lithography of Lithographie Galvanoformung Abformung ${ }^{8,9}$ and ultraviolet (UV) lithography ${ }^{10,11}$. These methods, however, are very time consuming and require advanced cleanroom facilities, making them cost prohibitive for general drug delivery. Thus, there is a need to explore new fabrication approaches that are low cost and can be carried out without expensive cleanroom facilities ${ }^{12,13}$. Here, we propose a simple three-step cleanroom-free method to make microneedles. In our method, preliminary microneedle molds are prepared by laser ablation, using readily available $\mathrm{CO}_{2}$ laser cutters. We used a specific two-dimensional (2D) geometric patterns consisting of lines that cross over each other at one point to ablate a sharp conical shape in an acrylic sheet. Our strategy to achieve high aspect ratio conical molds was to pass the laser beam via the same point several times while engraving (ablation), where each time the beam follows a different path. We used our technique to fabricate microneedle molds with different dimensions (depth, shape and tip angle). Later on, we used the molds to fabricate degradable microneedle patch with polyvinyl alcohol (PVA). PVA is a water-soluble polymer with wide practical application in a variety of fields, including wound dressing, implants, cell encapsulation, drug-delivery systems, soft contact lenses and dental applications, due to its excellent chemical resistance and physical properties, low toxicity and high biocompatibility ${ }^{14-16}$. An aqueous solution of PVA can form hydrogels through cast-drying process, where PVA physically cross links during the dehydration process ${ }^{17}$.

In summary, the proposed approach facilitates facile, ondemand and cleanroom-free fabrication of microneedles with a desired geometry.

\section{MATERIALS AND METHODS} Microneedle mold fabrication

The method used to create microneedles mold is shown in Figure 1a. Our strategy was to use a 2D drawing to fabricate a 3D

\footnotetext{
${ }^{1}$ Department of Electrical and Computer Engineering, Nano Lab, Tufts University, Medford, MA 02155, USA and ${ }^{2}$ Department of Biomedical Engineering, Tufts University, Medford, MA 02155, USA

Correspondence: Sameer Sonkusale (sameer@ece.tufts.edu)

*These authors contributed equally to this work.

Received: 2 June 2017; revised: 4 August 2017; accepted: 30 August 2017
} 
structure. Here, the microneedle mold was engraved on a commonly used clear cast acrylic sheet (part number of 8560K359. McMaster-Carr) using a $\mathrm{CO}_{2}$ cutter (Boss LS-1416) with maximum power of $60 \mathrm{~W}$. The FTIR transmission spectrum ${ }^{18}$ of the clear cast acrylic sheet shows that it has reasonable absorption around $10.6 \mu \mathrm{m}$ wavelength of the $\mathrm{CO}_{2}$ laser in fact responsible for the cutting of the sheet. Initially, laser beam was auto-focused on the surface of acrylic sheet. The fabrication starts by engraving lines that overlap only at their center cross-point. Each line was engraved with the same laser power and engraving speed, which results in almost the same depth per run. However, the engraving depth at the cross-point is higher since this point is traversed multiple times; the depth is then proportional to the number of times that the laser beam passes through the point (Figure 1a). As the laser beam passes the cross-point, a sharp cone starts to shape in the middle, which eventually leads to the formation of microneedle mold at the center of design (cross-point). The depth of the sharp cone can simply be adjusted by changing the number of lines used in the initial 2D CAD drawing. Here, we refer to our method as crossover lines (COL) laser ablation technique.

The fabrication process followed to fabricate microneedles is shown in Figure 1b. The engraved acrylic mold was washed with isopropanol and distilled water to remove the dust and particles from the surface and engraved areas. A nitrogen gun was used to remove the excess water on the surface. The mold was then dried in an atmospheric oven at $80^{\circ} \mathrm{C}$ for $30 \mathrm{~min}$. In the next step, the mold's replicate was created by casting Dow Corning polydimethylsiloxane (PDMS; Auburn, MI, USA) (with the ratio of 10:1 weight ratio of elastomer to curing agent) on the acrylic sheet. The PDMS-casted sheet was degassed and subsequently cured in the oven at $80^{\circ} \mathrm{C}$ for $2 \mathrm{~h}$. The PDMS microneedles were detached from the acrylic mold and were treated with oxygen plasma to activate the surface of PDMS. The microneedles were then silanized with trichloro $(1 \mathrm{H}, 1 \mathrm{H}, 2 \mathrm{H}, 2 \mathrm{H}$-perfluorooctyl) silane (from Sigma-Aldrich, Natick, MA, USA) under vacuum in a desiccator overnight ${ }^{19}$. Briefly, the sample was placed in a desiccator and $10 \mu \mathrm{L}$ of the silane solution was dispensed on a piece of aluminum foil in the desiccator. The desiccator was then vacuumed and was kept in that condition overnight to complete the process. PDMS (with the ratio of 10:1) was casted (to create a mold) on the silanized microneedles followed by degassing and curing in an atmospheric oven. The silane layer creates a barrier between PDMS microneedles and PDMS mold, avoiding them from bonding to each other, and facilitates their detachment. The final PDMS mold was used to create microneedles from different polymers. Methods used for fabrication of polymer-based microneedles are described in the "RESULTS AND DISCUSSION" section.

\section{RESULTS AND DISCUSSION}

\section{Characterization of the fabrication procedure}

Since we used laser cutter for microneedle fabrication, characterization of the cutter is important to achieve the insight on the shape and size of microneedles. Figure $2 a$ shows the engraved depth on an acrylic substrate for different laser power and speed settings. To fabricate the acrylic mold, we chose two different speed settings of 5 and $10 \mathrm{~mm} \mathrm{~s}^{-1}$. Later on, we evaluated the effect of laser power on the engraved depth (with $1 \%$ resolution). The cutter starts to engrave the acrylic surface at power levels greater than $5.4 \mathrm{~W}$, which is $9 \%$ of our laser cutter's maximum power setting $(60 \mathrm{~W})$, and for levels below $5.4 \mathrm{~W}$, no engraving was observed. Therefore, $5.4 \mathrm{~W}$ laser power (for previously mentioned speeds) was chosen to achieve fine engraving resolution and used for all fabricated microneedle acrylic molds in this paper. Microneedles of different shape, size and angle were fabricated by altering three parameters of laser scanning speed, number of lines used to fabricate each needle and the length of the lines (Figure 2b). The effect of these parameters on height and tip angle of the PDMS microneedles (fabricated using the acrylic mold) is quantified and depicted in Figure 2c. On the basis of our primary study, we considered two different levels for the length of the lines ( 1 and $1.5 \mathrm{~mm}$ ) and engraving speed ( 5 and $10 \mathrm{~mm} \mathrm{~s}^{-1}$ ) which are elaborated in Table 1. Five levels were also considered for number of lines used to fabricate the acrylic mold, which are 4, $6,8,10$ and 12 . We observed that the length of lines does not affect the height of needles considerably; however, longer lines a

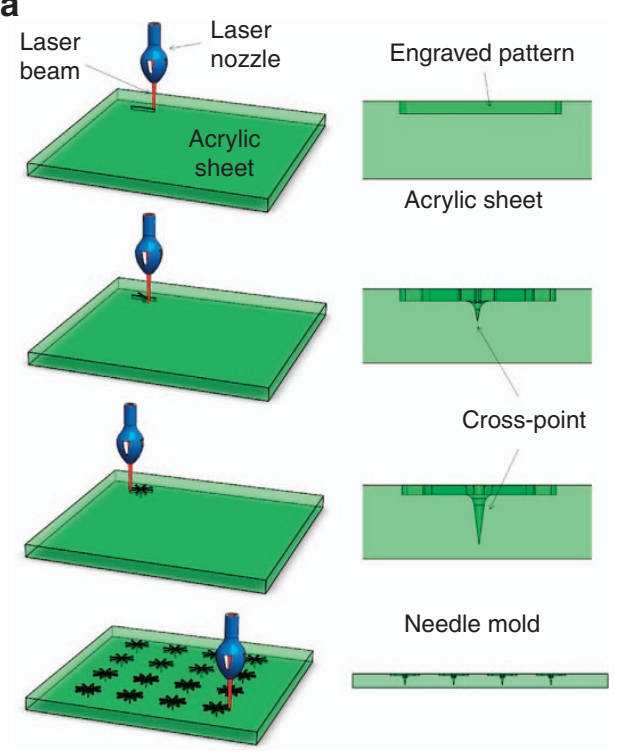

b

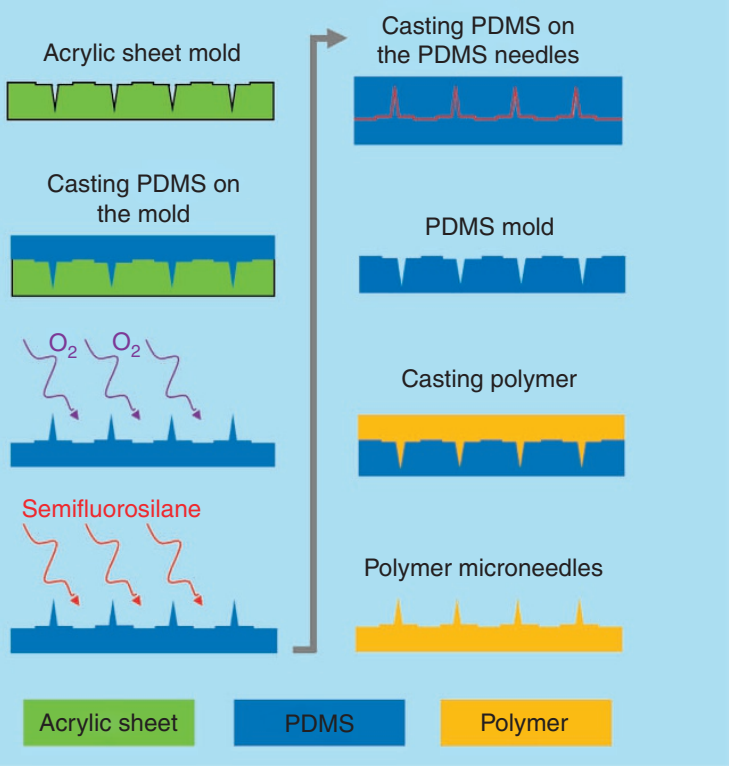

Figure 1 Fabrication of microneedle mold. (a) $\mathrm{CO}_{2}$ laser cutter was used to fabricate microneedle acrylic mold using the proposed cross-over lines (COL) technique, (b) the acrylic mold was used to fabricate polydimethylsiloxane (PDMS) microneedles mold, which can be used to fabricate a variety of polymer-based microneedles. 

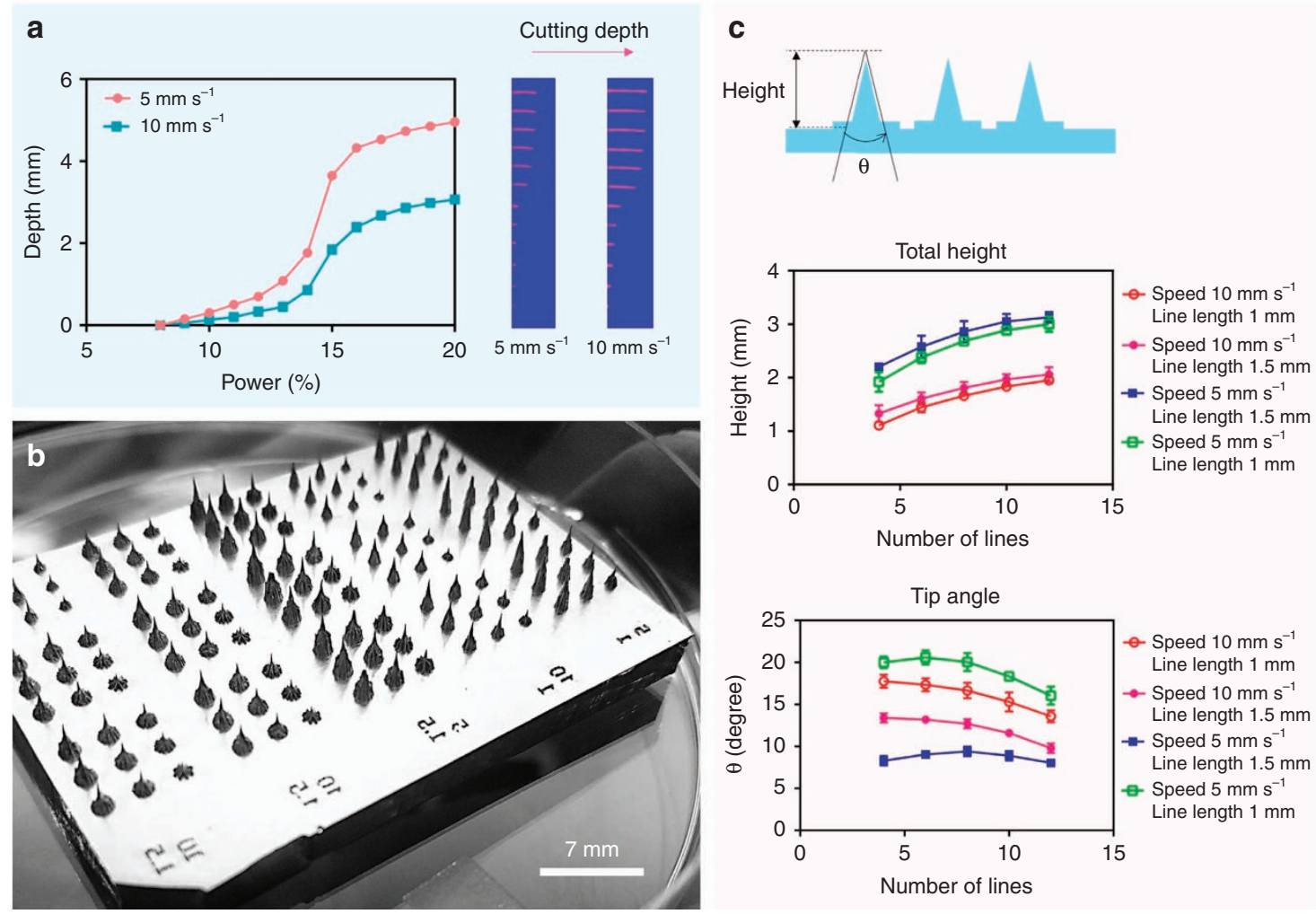

Figure 2 (a) The depth of the engraving with certain power and speed of $\mathrm{CO}_{2}$ laser, (b) different size of needles resulted from different settings of laser power and speed and (c) different height and tip angle of the needles, depending on the speed (first number in the legend) and length of lines in millimeter (second number in the legend after dash). Error bar plot extracted from 10 different samples.

Table 1 Needle types based on fabrication parameters, including engraving speed and length of the lines in COL design

\begin{tabular}{lccc}
\hline Microneedle type & $\begin{array}{c}\text { Engraving speed } \\
\left(\mathrm{mm} \mathrm{s}^{-1}\right)\end{array}$ & $\begin{array}{c}\text { Length of lines } \\
(\mathrm{mm})\end{array}$ & Power (W) \\
\hline $\mathrm{MN}-1$ & 5 & 1.0 & 5.4 \\
$\mathrm{MN}-2$ & 5 & 1.5 & 5.4 \\
$\mathrm{MN}-3$ & 10 & 1.0 & 5.4 \\
$\mathrm{MN}-4$ & 10 & 1.5 & 5.4 \\
\hline
\end{tabular}

Abbreviation: $\mathrm{COL}$, crossover lines.

lead to slightly sharper needles, which is most probably due to more stable laser beam at the crosspoint for longer lines. Depending on the application of the needle, specific size of the needle is required. Height and tip angle are two major characteristics of the microneedles. These two factors, which are discussed and characterized in Figure $2 c$, can be readily controlled by either changing the number of lines or engraving speed.

\section{Microneedle shape characterization}

To create a better intuition about the shape of the fabricated microneedles using different above-mentioned factors, we captured and quantified the profile of the fabricated needles using image processing (Figure 3 ). Figure 3 shows the shape of the resulted microneedles, where the first column shows the microneedles fabricated with $1 \mathrm{~mm}$ engraved lines and the second column shows the needles with $1.5 \mathrm{~mm}$ length of lines. As discussed earlier, they do not differ in depth (or height of the needle) to a large extent due to the fact that the height of the needles are mostly dependent on the number of engraved lines. However, the length of the lines affect the overall shape of the needles where longer lines create sharper conical shape. Observably, needles with higher height are achievable by simply increasing the number of lines to fabricate each needle.

\section{Fabrication of fine microneedles}

Needles of different heights and tip angles can be fabricated with the proposed technique. As it is clear in Figure 4a, fabricated microneedles have two parts of a head and a base. The head has a fine conical shape suitable for microneedle applications; however, the base of the fabricated needles has a star shape, which is not useful for such applications. In order to separate these two parts, and just use the head to create the microneedle mold, two sheets of acrylic were placed on top of each other; bottom sheet was $5.5 \mathrm{~mm}$ and the top sheet was $1.5 \mathrm{~mm}$ clear cast acrylic sheet. Subsequently, laser cutter is used to engrave the sheets using the $\mathrm{COL}$ technique explained in "Microneedle mold fabrication" section (shown also in Figure 1). Here, we slightly increase the engraving power up to $12 \%$ of the maximum power to compensate for the additional sheet. The engraved acrylic substrates are shown Figure $4 \mathrm{~b}$. The top sheet is chosen to be thin enough to contain only the base part (Figure $4 \mathrm{~b}$ ). Therefore, the bottom sheet only contains the head portion of the microneedles (Figure 4b). These two sheets were then separated from each other using a razor blade (the sheets are weakly attached to each other at engraving points). The bottom sheet is used to fabricate the PDMS microneedles (Figure 4c). This way, a needle with a fine conical shape is achieved (Figure 4c). The same procedure can be used to control the height and angle of the fabricated needles 


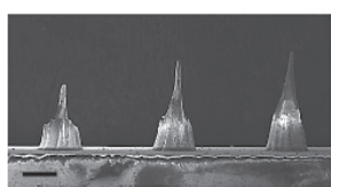

$\mathrm{MN}-1$

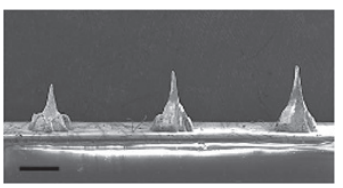

$\mathrm{MN}-3$

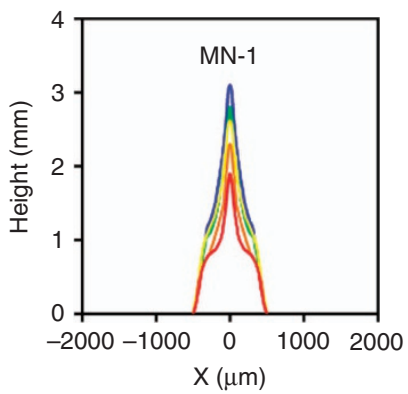

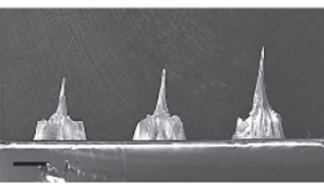

$\mathrm{MN}-2$

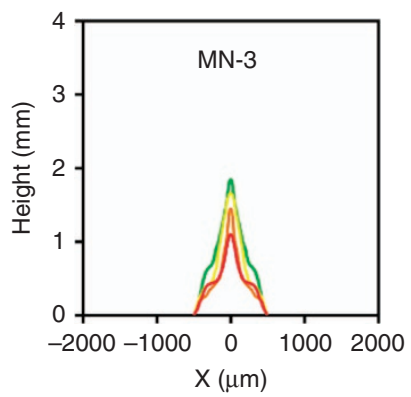

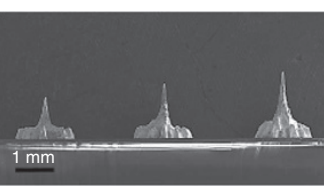

$\mathrm{MN}-4$
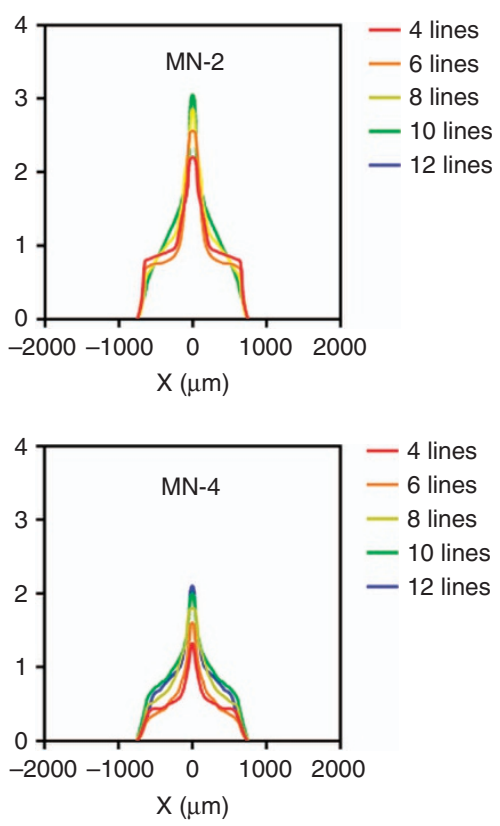

Figure 3 Shape of the microneedles with different lengths and number of lines. Scanning electron microscopic (SEM) images (from left to right) are for needles fabricated with 4, 6 and 8 lines.
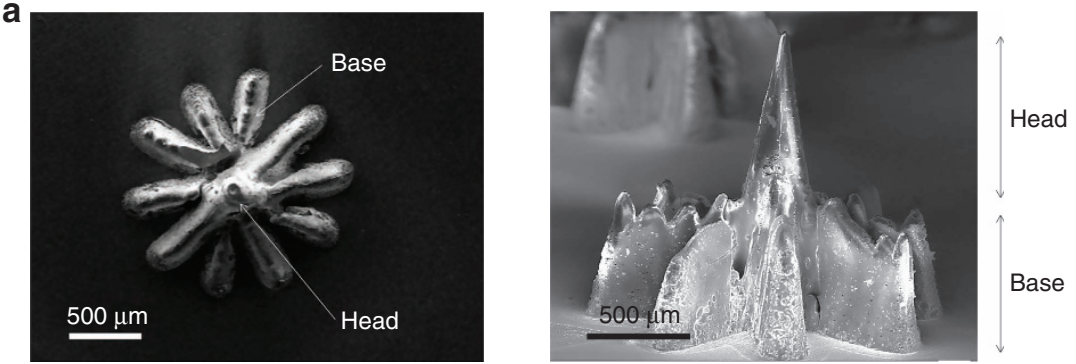

b

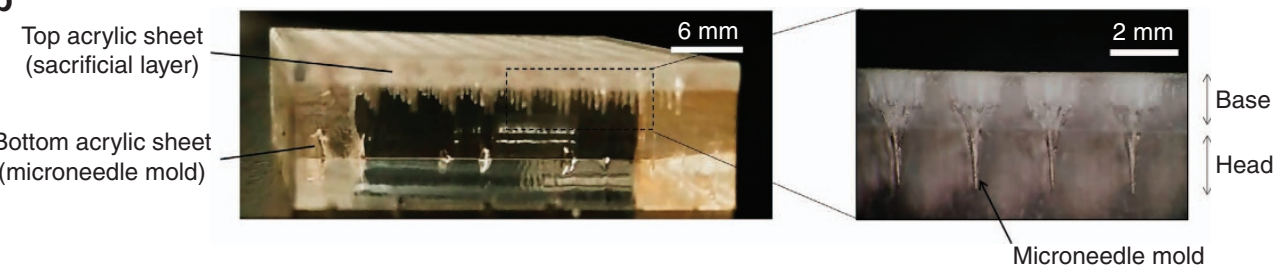

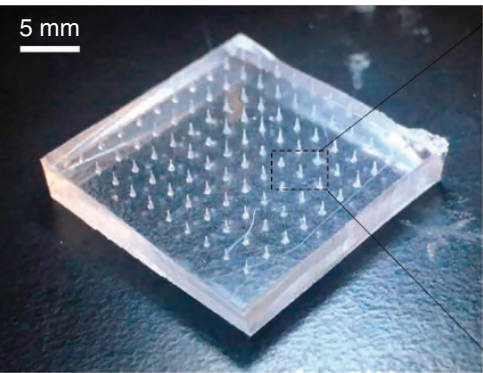

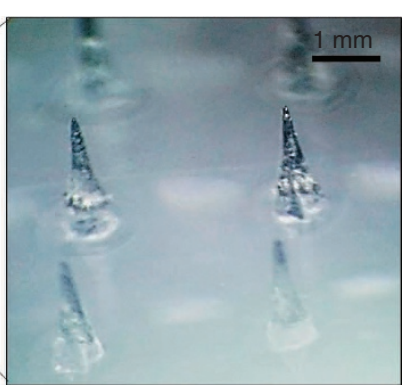

Fine microneedles d

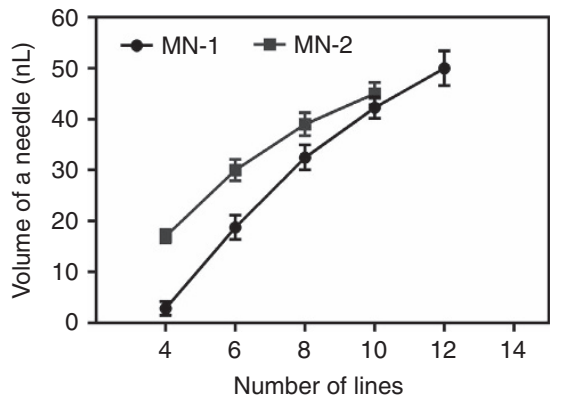

Figure 4 Fabrication of fine microneedles. (a) Fabricated microneedles showing head and base parts, (b) a sacrificial method was used to remove the base of the needles and create mold to fabricate fine microneedles, (c) fine fabricated PDMS microneedles fabricated out of acrylic mold and (d) calculated volume of fine needles based on mold fabrication parameters. 


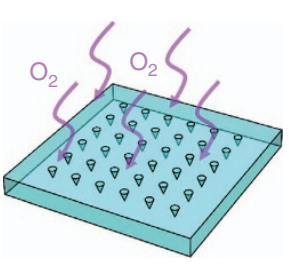

b

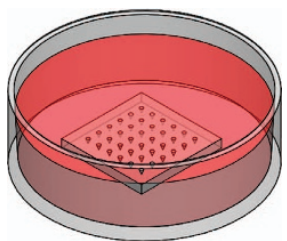

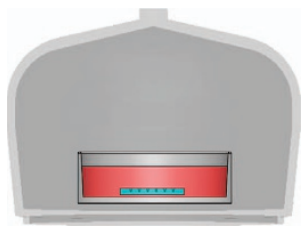

d

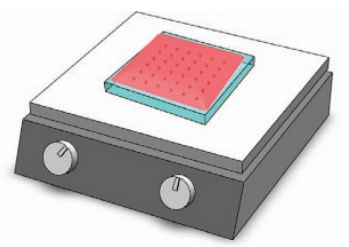

e

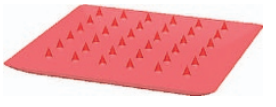

f

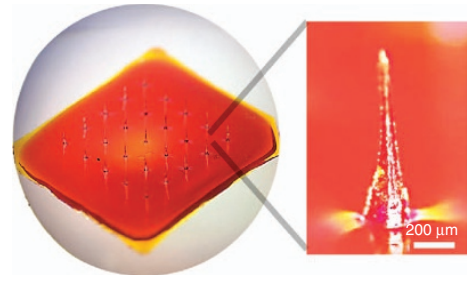

h

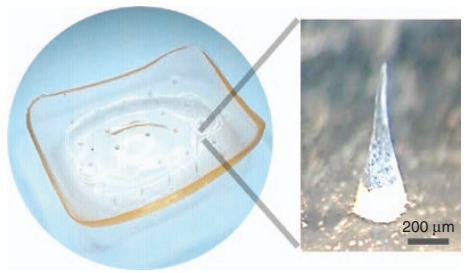

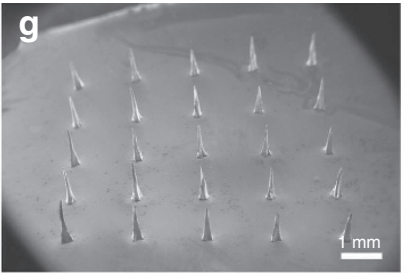
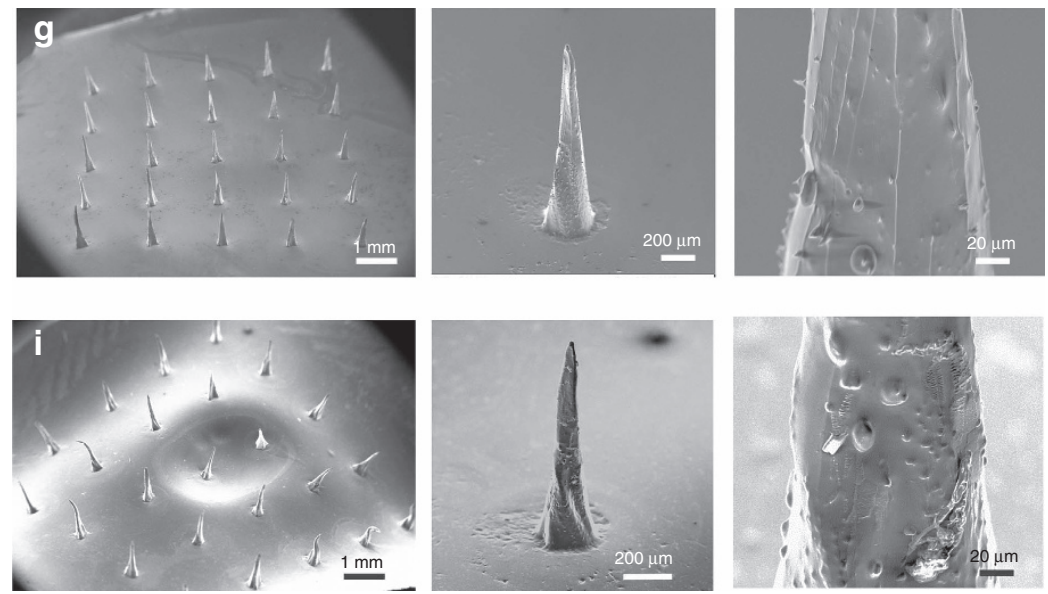

j

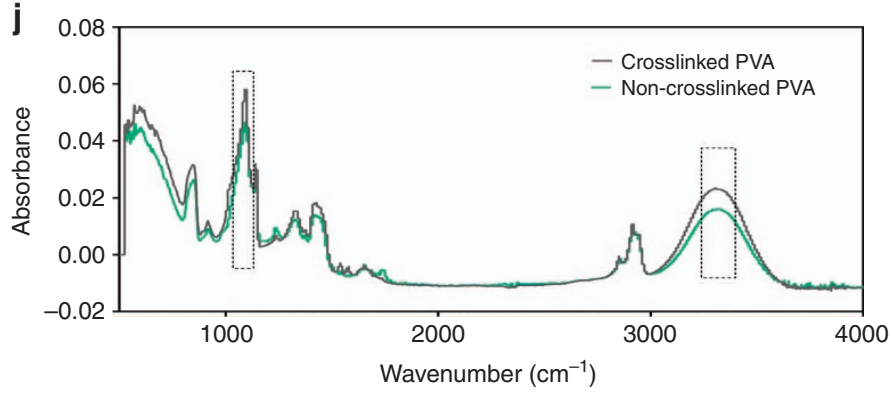

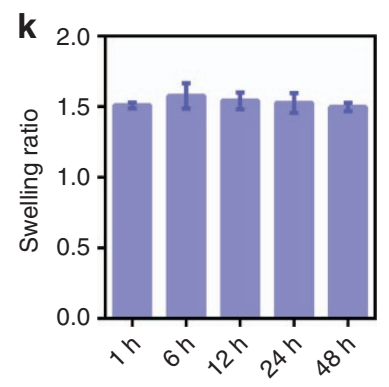

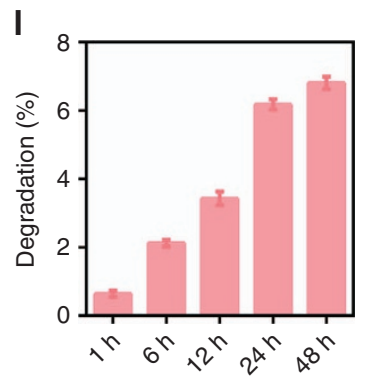

m

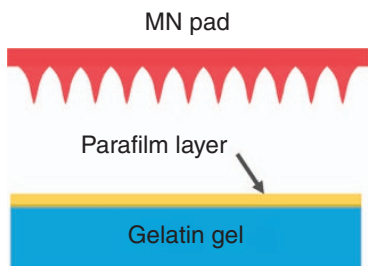

\section{n}

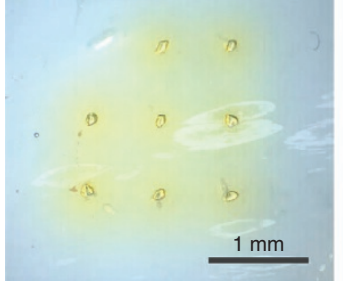

o

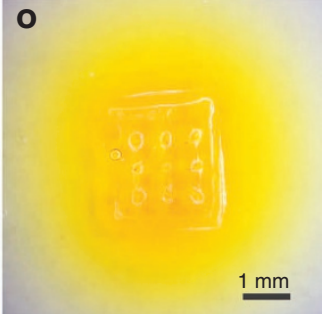

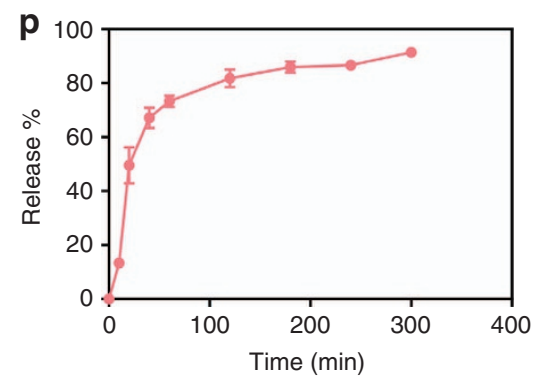

Figure 5 Polymer casting and microneedle fabrication method and needle characterization. (a) Initially the surface of the PDMS mold in oxygen plasma treated; (b) the mold is submerged in petri dish with the polyvinyl alcohol (PVA) solution containing phenol red dye (as a drug model); (c) air bubbles were removed and the solution was condensed by placing the dish in vacuum condition inside desiccator for 7 days; (d) the mold was placed on hot plate to dehydrate and dry the patch; (e) the microneedle patch was pilled off the mold after complete drying; (f) the fabricated PVA microneedle patch, the red color is due to phenol red; (g) scanning electron microscopic (SEM) images of the PVA needle before drug release, showing smooth dense surface with not observable porosity; (h) PVA microneedle patch, after drug release (incubation in phosphate-buffered saline (PBS) at $37^{\circ} \mathrm{C}$ for $24 \mathrm{~h}$ ), the red dye is almost gone but the needles almost preserve their shape; (i) SEM images of needle after release (dried); shape and surface of the needles did not significantly change, confirming that the drug release mostly happens due to diffusion and not degradation; (j) FTIR results for non-crosslinked and crosslinked PVA (PVA microneedle patch); (k) swelling ratio of the PVA needles; (I) degradation of the PVA needle in PBS (less than $8 \%$ after $48 \mathrm{~h}$ ) (m) showing the in vitro skin and release model; (n) release of the drug into the hydrogel after $5 \mathrm{~min}$; (o) release of the drug after $5 \mathrm{~h}$ and $(\mathbf{p})$ cumulative release model over time.

(explained before). The volume of each microneedle is an important metric for drug delivery.

We calculated the volume of each needle based on the mold fabrication parameters (Figure $4 \mathrm{~d}$ ). This will eventually help to estimate the amount of drug that each needle caries.

\section{COL versus other drilling methods}

There are in fact different ways of drilling by laser, which are single shot drilling, trepanning and helical drilling as shown in Supplementary Figure S1. We discuss how these methods work and also compare the advantage of COL method to those other 
methods. One of the basic methods for drilling is single shot drilling which is shown in Supplementary Figure S1a. This method creates a hole on the surface by focusing the laser beam at a point and pumping the laser power in pulsed mode. This process makes a cylindrical and blunt hole which is rather really narrow. Single shot drilling never yields a conical hole where the base diameter is larger than the tip diameter, not to mention that there is no control on the base diameter of the needle. COL method on the other hand allows implementation of needles with different base diameters such as 1.5 and $1 \mathrm{~mm}$ of base diameter as shown in Figures 2 and 3.

The other method of drilling is known to be trepanning shown in Supplementary Figure S1b. Although this is a good way of drilling but all it results is a wider hole with user-defined diameter. However, there is no 3D shaping of the hole structure, such as the formation of conical shape. The advantage of COL method is that it can translate a 2D design to a 3D structure, but trepanning is unable to do this. Helical drilling is shown in Supplementary Figure S1c; it also suffers from the same disadvantages of the trepanning drilling. We were also able to fabricate microneedle patches with different sizes. A patch of $6 \times 6 \mathrm{~cm}^{2}$ is shown in Supplementary Figure S3.

\section{Polymer microneedles fabrication}

Degradable microneedles were fabricated using PVA polymer (from Sigma-Aldrich). The fabrication procedure followed is shown in Figures 5a-e. Initially a solution of $10 \%$ PVA was prepared by mixing PVA powder in distilled water. As a drug model, phenol red pH dye powder (from Sigma-Aldrich) was added to the mixture to create $0.1 \%$ (weight/volume) concentration. The mixture was placed in oven at $50^{\circ} \mathrm{C}$ for $5 \mathrm{~h}$ to create a homogeneous solution. Next, the microneedle PDMS mold was oxygen plasma treated for $5 \mathrm{~min}$. Plasma treating creates a hydrophilic surface that facilitates penetration of PVA (10\%) solution into PDMS microneedles mold cavities (Figure 5a). The mold was then submerged in the prepared PVA solution (Figure $5 b$ ), and the sample was placed in a desiccator under vacuum condition for 7 days (Figure $5 c$ ). This allows the trapped air bubbles inside the mold cavities to come out and be replaced by PVA solution. Vacuum condition (for 5 days) also removes water moisture slowly and creates a dense PVA solution. Other possible approach for avoiding excessive time of 7 days would be to use a vacuum oven that could remove the bubbles faster and make the solution dense in a few hours. Moreover, if the drug is such that it does not degrade at slightly elevated temperatures, one could also apply heat to speed up the densification process. The mold was then placed on a hot plate at $40^{\circ} \mathrm{C}$ to dry out and crosslink the PVA patch (Figure $5 \mathrm{~d}$ ). After $5 \mathrm{~h}$, the PVA microneedle patch gets hard enough to be removed from the microneedle mold (Figure 5e). It is worth mentioning that the final drying step at $40^{\circ} \mathrm{C}$ can be replaced by drying the microneedle patch in a dry desiccator at room temperature for 2 days in case the drug is sensitive to elevated temperature.

The prepared PVA microneedle with this method showed adequate physical stability (Figure $5 f$; the red color is due to phenol red). SEM images taken from the samples show that the PVA needles have a smooth surface with minimum roughness and very low porosity (Figure $5 \mathrm{~g}$ ). This shows the dense structure of the PVA with minimum air trapped inside the structure. To examine the drug release mechanism, the microneedle patch was immersed in phosphate-buffered saline (PBS from Sigma-Aldrich) solution and placed inside an incubator. We observed that phenol red dye, which serves here as the drug model, gets released from the patch almost completely after one day and the patch becomes clear (Figure $5 \mathrm{~h}$ ). However, the needles preserve their structure for several days in PBS (Figures $5 \mathrm{~h}$ and i). The increase in crystallization degree and hydrogen bonding are the main reasons for the low degradation rate of PVA microneedle patch (Figure 5h). The degree of crystallization depends on the drying conditions. Slow drying at room temperature allows the slow formation of crystal ${ }^{20,21}$. FTIR spectrum was acquired for PVA microneedle patch and compared with non-crosslinked PVA
(Figure 5j). An increase in the intensity of $1143 \mathrm{~cm}^{-1}$ peak is observed for PVA microneedle patch compared to non-crosslinked PVA, which is an indicator of the increase in the degree of crystallinity in physically crosslinked PVA ${ }^{22}$. Moreover, the dehydration of free water brings polymer chains close to each other due to the formation of additional hydrogen bonds (increase in the intensity of hydroxyl group, FTIR (Figure 5j)), and this provides enough crosslink points that facilitate the formation of polymer networks ${ }^{22}$. The swelling and degradation tests were also carried out for the fabricated PVA microneedles (Figures $5 \mathrm{k}$ and I). We immersed the weighed dry PVA microneedle patch in the release medium $(\mathrm{PBS}, \mathrm{pH}=7.4)$ at $37^{\circ} \mathrm{C}$ for $1,6,12,24$ and $48 \mathrm{~h}$. At regular intervals, the swollen samples were wiped gently with paper and weighed, then samples were dried in room temperature to reach constant weight and then brought back to PBS. The swelling degree and the degradation rate were calculated using Equations 1 and 2 below ${ }^{23}$ :

$$
\begin{aligned}
& \text { Degree of swelling }=\frac{M-M_{i}}{M_{i}} \times 100 \\
& \text { Degradation rate }=\frac{M-M_{d}}{M_{i}} \times 100
\end{aligned}
$$

where $M$ is the weight of swollen sample, which was wiped with paper, $M_{d}$ is the dried mass of immersed sample in PBS and $M_{i}$ is the initial dry mass of the sample.

Also, another important metric is the drug capacity of the microneedle patch. This depends on the volume of each microneedle, which depends on the fabrication parameters of the mold and the nature of polymer (Figure $4 d$ ). If the initial mixing ratio (mass/mass) of the drug to polymer and the density of the polymer and the drug is known, the amount of drug carried by each needle can be easily determined. Finally, the number of needles in each patch will determine the total dose of delivered drug.

\section{In vitro drug release}

We used gelatin hydrogel derived from bovine skin (from SigmaAldrich) as a model tissue for drug release studies, which is optically transparent and has similarity to the skin tissue. We placed gelatin $10 \%$ in the refrigerator for solidification. Then, we placed the polymer membrane (Parafilm M, Bemis ${ }^{\circledR}$ Neenah, WI, USA) on top of the hydrogel surface (Figure $5 \mathrm{~m}$ ). A microneedle patch of $1 \times 1 \mathrm{~cm}^{2}$ containing phenol dye was then pressed against the membrane surface to insert the needles into the gelatin hydrogel (Figure $5 \mathrm{~m}$ ). The polymer membrane acted as a diffusion barrier and prevented the abrupt release of drug from microneedle's bulk PVA substrate into the underlying gelatin hydrogel. The phenol red was used as the model drug, and the standard curve was drawn to quantify the drug release. At each time stamp, the microneedle patch is detached from the hydrogel to stop further release (Figures $5 n-p$ ). By increasing the temperature, gelatin liquified and allowed the measurement of the phenol red concentration released using UV-VIS spectroscopy at $450 \mathrm{~nm}$ wavelength. Almost half of the encapsulated phenol red inside microneedle was released in $20 \mathrm{~min}$ and the remaining was released in the next $5 \mathrm{~h}$ (Figures $5 \mathrm{n}-\mathrm{p}$ ). The test is performed at room temperature. As most of the drug has been released in the first hour, PVA microneedles can be removed and disposed from the skin in a real scenario.

Drug release is governed either by the drug diffusion through hydrogel or by the degradation of the hydrogel. We performed swelling and degradation test by incubating PVA microneedle patch in PBS for $48 \mathrm{~h}$ at $37^{\circ} \mathrm{C}$ to understand the mechanism of drug release from PVA (Figures $5 \mathrm{k}$ and I). According to the degradation test result, only $6.81 \%$ of the PVA degraded during $48 \mathrm{~h}$ (Figure 5l); thus, polymer erosion did not play a significant role in drug release from PVA. However, the high hydrophilic property of PVA leads to high absorption of water and consequently, fast diffusion of drug in release media ${ }^{24}$. Previous studies showed that the drug release from microneedles 
prepared by mixing polymer and drug directly is controlled by diffusion. In diffusion-controlled release, the molecular size and weight play an important role. Phenol red is considered as the small molecule, and its fast release can be explained by diffusion controlled release theory. After incubation of PVA microneedle in PBS, the free water penetrates into the empty regions of the polymer network till the equilibrium state is reached. Swelling of PVA continued for about $1 \mathrm{~h}$ until the osmotic pressure equals the forces of the crosslinking bonds that maintain the structure of the polymer network stable. At the time these two forces gets equal, no further water gain is observed ${ }^{25}$.

PVA microneedles were shown here as a representative example. However, we also fabricated microneedles with different polymers including photo curable resin (from Formlabs Inc. Somerville MA), polycaprolactone (PCL) and chitosan (both from Sigma-Aldrich). Please see Supplementary Section (Supplementary Figure S2), which also includes the fabrication steps for these polymers. It is worth noting here that PCL and chitosan microneedles are biodegradable.

\section{CONCLUSION}

We showed a simple, inexpensive cleanroom-free approach for making high aspect ratio microneedles using molds fabricated via laser ablation. Laser ablation of acrylic substrate using $\mathrm{CO}_{2}$ laser cutting machine in a COL pattern is shown to create 3D conical molds for subsequent microneedle fabrication. The COL approach relies on the fact that each engraving line results in almost the same depth per run for same laser power and engraving speed. When lines cross, the engraving depth at the cross-point is higher since this point is traversed multiple times, resulting in a sharp cone resembling the conical microneedle mold at this crossover point. PDMS microneedles were fabricated by casting the PDMS on the mold. The PDMS microneedles were then reused to fabricate a PDMS replica mold using silanizing and PDMS casting. None of the steps used to fabricate the microneedle mold based on the proposed methodology requires cleanroom facility or any complicated and expensive procedure. We characterized the needles based on their height and tip angle so that variety of needles with different heights and tip angles can be achieved by simply changing the length and number of lines of the initial 2D design. The process is reproducible and can facilitate fabrication of microneedles at industrial scale for many biomedical applications, such as gene therapy and proteinbased drug delivery applications. For proof of concept, the PDMS microneedle mold prepared with the proposed technique is used to fabricate degradable microneedle patch based on PVA. Microneedles made from $\mathrm{PCL}$, chitosan and other polymers were also explored. We also showed the release model of the PVA microneedle array to demonstrate its suitability for drug delivery applications. Future investigations will include animal studies for transdermal drug delivery using these microneedles for a variety of drugs.

\section{ACKNOWLEDGEMENTS}

We acknowledge the partial support of the Office of Naval Research (ONR) (Grant No. N0014-16-1-2550).

\section{COMPETING INTERESTS}

The authors declare no conflict of interests in this work.

\section{REFERENCES}

1 Prausnitz MR, Langer R. Transdermal drug delivery. Nature Biotechnology 2008; 26: 1261-1268.

2 Marwah H, Garg T, Goyal AK et al. Permeation enhancer strategies in transdermal drug delivery. Drug Delivery 2016; 23: 564-578.
3 Foldvari M, Chen DW, Nafissi N et al. Non-viral gene therapy: Gains and challenges of non-invasive administration methods. Journal of Controlled Release 2016; 240 : 165-190.

4 Larrañeta E, Lutton REM, Woolfson AD et al. Microneedle arrays as transdermal and intradermal drug delivery systems: Materials science, manufacture and commercial development. Materials Science and Engineering: R: Reports 2016; 104: 1-32.

5 Chen W, Li H, Shi D et al. Microneedles as a delivery system for gene therapy. Frontiers in Pharmacology 2016; 7: 137.

6 Lee I, Lin WM, Shu JC et al. Formulation of two-layer dissolving polymeric microneedle patches for insulin transdermal delivery in diabetic mice. Journal of Biomedical Materials Research_Part A 2017; 105: 84-93.

7 Packianather MS, Le CH, Pham DT et al. Advanced micro and nano manufacturing technologies used in medical domain. InIFMBE Proceedings 2016; 2016: 120-123.

8 Vinayakumar KB, Kulkarni PG, Nayak MM et al. A hollow stainless steel microneedle array to deliver insulin to a diabetic rat. Journal of Micromechanics and Microengineering 2016; 26: 65013.

9 Moon SJ, Lee SS, Lee HS et al. Fabrication of microneedle array using LIGA and hot embossing process. Microsystem Technologies 2005; 11: 311-318.

10 Sullivan SP, Murthy N, Prausnitz MR. Minimally invasive protein delivery with rapidly dissolving polymer microneedles. Advanced Materials 2008; 20: 933-938.

11 Kim K, Park DS, Lu HM et al. A tapered hollow metallic microneedle array using backside exposure of SU-8. Journal of Micromechanics and Microengineering 2004; 14: 597-603.

12 Hirai $Y$, Inamoto $Y$, Sugano $\mathrm{K}$ et al. Moving mask UV lithography for three-dimensional structuring. Journal of Micromechanics and Microengineering 2007; 17: 199-206.

13 Maldonado JR, Peckerar M. X-ray lithography: Some history, current status and future prospects. Microelectronic Engineering 2016; 161: 87-93.

14 Karimi A, Navidbakhsh M, Faghihi S. Fabrication and mechanical characterization of a polyvinyl alcohol sponge for tissue engineering applications. Perfusion 2014; 29: 231-237.

15 Oun R, Plumb JA, Wheate NJ. A cisplatin slow-release hydrogel drug delivery system based on a formulation of the macrocycle cucurbit [7] uril, gelatin and polyvinyl alcohol. Journal of Inorganic Biochemistry 2014; 134: 100-105.

16 Murakami T, Sakai N, Yamaguchi T et al. Evaluation of a superior lubrication mechanism with biphasic hydrogels for artificial cartilage. Tribology International 2015; 89: 19-26.

17 Noh T, Bando Y, Ota K et al. Tear force of physically crosslinked poly (vinyl alcohol) gels with different submicrometer-scale network structures. Journal of Applied Polymer Science 2015; 132: 1-6.

18 Duan G, Zhang C, Li A et al. Preparation and characterization of mesoporous zirconia made by using a poly (methyl methacrylate) template. Nanoscale Research Letters 2008; 3: 118-122.

19 Rezaei Nejad H, Goli Malekabadi Z, Kazemzadeh Narbat M et al. Laterally confined microfluidic patterning of cells for engineering spatially defined vascularization. Small 2016; 12: 5132-5139.

20 Mallapragada SK, Peppas NA, Colombo P. Crystal dissolution-controlled release systems. II. Metronidazole release from semicrystalline poly (vinyl alcohol) systems. Journal of Biomedical Materials Research 1997; 36: 125-130.

21 Mallapragada SK, Peppas NA. Crystal unfolding and chain disentanglement during semicrystalline polymer dissolution. AIChE Journal 1997; 43: 870-876.

22 Otsuka E, Suzuki A. Swelling properties of physically cross-linked PVA gels prepared by a cast-drying method. Progress in Colloid Polymer Science 2009; 136: 121-126.

23 Khoshakhlagh P, Rabiee SM, Kiaee G et al. Development and characterization of a bioglass/chitosan composite as an injectable bone substitute. Carbohydrate Polymers 2017; 157: 1261-1271.

24 Takamura A, Ishii F, Hidaka $\mathrm{H}$. Drug release from poly (vinyl alcohol) gel prepared by freeze-thaw procedure. Journal of Controlled Release 1992; 20: 21-27.

25 Pasparakis G, Bouropoulos N. Swelling studies and in vitro release of verapamil from calcium alginate and calcium alginate-chitosan beads. International Journal of Pharmaceutics 2006; 323: 34-42. 